\title{
Estructura genética en cinco especies de flebótomos (Lutzomyia spp.) de la serie townsendi, grupo verrucarum, en Colombia (Diptera: Prychodidae)
}

\author{
Claribel Hernández ${ }^{1}$, Manuel Ruiz-García ${ }^{2 *}$, Leonard Munstermann ${ }^{3}$ \& Cristina Ferro ${ }^{4}$ \\ 1. Secretaria de Salud de Cundinamarca, Bogotá DC, Colombia. \\ 2. * Correspondence: Grupo de Genética de Poblaciones Molecular y Biología Evolutiva (Unidad de Genética). \\ Departamento de Biología. Facultad de Ciencias. Pontificia Universidad Javeriana. Cra 7A No 43-82. Bogotá DC. \\ Colombia.mruiz@javeriana.edu.co \\ 3. Yale University, New Haven, Connetticutt, EEUU. \\ 4. Instituto Nacional de Salud. Bogotá DC. Colombia.
}

Recibido 08-XII-2007. Corregido 30-VI-2008. Aceptado 31-VII-2008.

\begin{abstract}
Genetic structure in five Phlebotominae (Lutzomyia spp.), townsendi series, verrucarum group, in Colombia (Diptera: Prychodidae). Sixteen isoenzyme patterns were analyzed for five Colombian Lutzomyia species. The average unbiased expected heterozygosity levels ranged from 0.098 (Lu. youngi) to 0.215 ( $L u$. torvida). The five species samples, taken all the isoenzymes employed, were significantly deviated from the Hardy-Weinberg equilibrium by homozygous excess with classical as well as Markov chain exact tests. Possible causes: (1) Wahlund effect within populations due to subdivision and/or sampling. Endogamy could be discarded because these loci were affected by highly different levels of homozygous excess. (2) Null alleles could be not discarded, at least for some isoenzymes. The hierarchical Wright's F analysis showed high and significant values for each parameter. The average $\mathrm{F}_{\mathrm{IT}}$ value was 0.655 with a conspicous homozygous excess at a global level (all species taken together); the average $\mathrm{F}_{\mathrm{IS}}$ value was significantly positive $(0.515)$ as well, with homozygous excess within each species. The genetic heterogeneity between the fives species was noteworthy $\left(\mathrm{F}_{\mathrm{ST}}=\right.$ 0.288), indicating clear genetic differentiation. The more related species pairs were Lu. longiflocosa-Lu. torvida (0.959) and Lu torvida-Lu. spinicrassa (0.960); while Lu. torvida-Lu. youngi (0.805) and Lu. quasitownsendiLu. youngi (0.796) were the most divergent (Nei's genetic identity matrix). UPGMA and Wagner algorithms showed that the most divergent species was Lu. youngi, whereas the most related were Lu. longiflocosa-Lu. torvida and Lu torvida-Lu. spinicrassa. A spatial autocorrelation analysis (Moran's I index) revealed a very weak, or inexistent spatial structure, which means that the speciation events between these species were independent from the geographic distances from where they currently live. Rev. Biol. Trop. 56 (4): 1717-1739. Epub 2008 December 12 .
\end{abstract}

Key Words: Isoenzymes, population genetics, Lutzomyia, verrucarum group, Colombia.

Las leishmaniosis son zoonosis causadas por parásitos protozoos intracelulares obligados del género Leishmania. La forma infectante, promastigote, es inoculada al ser humano a través de la picadura de un insecto vector del género Lutzomyia (Subfamilia Phlebotominae), durante la ingesta sanguínea. Estas enfermedades actualmente prevalecen en cuatro continentes. Son consideradas endémicas en 88 países. Aproximadamente, 350 millones de personas viven en estas áreas. Sólo 32 países tienen notificación obligatoria. En realidad, un número substancial de casos nunca se reporta. De los 1.5-2 millones de nuevos casos que se estiman que ocurren anualmente, solo 600000 se reportan oficialmente. La distribución geográfica de las leishmaniasis está limitada por la distribución de los flebótomos (Clase Insecta, Orden Diptera, Suborden Nematocera, Familia Prychodidae, Subfamilia Phlebotominae). Su susceptibilidad a climas fríos, sus preferencias alimenticias y su capacidad para soportar el 
desarrollo interno de especies especificas del parásito los hace excelentes propagadores de la enfermedad (WHO 2000, 2002). Las leishmaniosis presentan una variedad de manifestaciones clínicas que difieren ampliamente en severidad y en su impacto sobre la salud humana. La infección puede cursar de manera asintomática, producir discretas lesiones en la piel o por el contrario, destruir la mucosa orofaríngea, infiltrar el bazo y la médula ósea produciendo una enfermedad sistémica que lleva a la muerte.

De las 500 especies de flebótomos conocidas, solo 30 de ellas han sido identificadas como vectores de la enfermedad (WHO 2000, 2002). El género Lutzomyia contiene la mayoría de los Phlebotominae americanos con cerca de 400 especies divididas en numerosos subgéneros, grupos y series. Se considera el grupo verrucarum como uno de los grupos de flebotomineos neotropicales más importantes en salud pública, con 42 especies divididas en cuatro series: serrana (10 spp), verrucarum (15 spp), pia (8 spp) y townsendi (9 spp). Diez de estas especies están señaladas como importantes vectores potenciales y confirmados de Leishmania spp y Bartonella bacilliformis. La serie townsendi conformada por nueve taxones, presenta siete especies en Colombia (Bejarano et al. 2003), asociadas con la leishmaniosis cutánea y mucocutánea en focos endémicos donde Leishmania (Viannia) braziliensis (Lainson 1983) es el principal agente etiológico. Las especies presentes en Colombia son $L u$. townsendi, Lu. sauroida, Lu. longiflocosa, Lu. torvida, Lu. spinicrassa, Lu. quasitownsendi y Lu. youngi. Las cinco últimas son objeto de estudio del presente trabajo presentando una distribución altamente endémica, especialmente abundante en áreas montañosas y bien adaptadas a los cultivos de café, tanto tradicionales como tecnificado. Lu. longiflosa, Lu. torvida y $L u$. quasitownsendi poseen una distribución exclusivamente colombiana, mientras que $L u$. spinicrassa se localiza también en Venezuela y Lu. youngi en Colombia, Venezuela y Costa Rica. Las hembras de esta serie son picadoras agresivas, altamente antropofílicas (Young
1979), marcadamente similares en estructura morfológica (isomórficas) y es difícil identificarlas sin asociarlas con los machos (Young 1979, Feliciangeli y Murillo 1987, Kreutzer et al. 1990), caracterizados por una espina basal aislada y tres espinas dístales en el estilo de la terminalia (Feliciangeli y Murillo 1987, Kreutzer et al. 1990).

Para separar esas especies de flebótomos se han utilizado varias metodologías: isoenzimas (Kreutzer et al. 1990), amplificación al azar de ADN polimórfico (RAPD; Adamson et al. 1991,1993), hidrocarburos cuticulares e hibridación de ADN (Maigon et al. 1993), estructura coriónica (Feliciangeli et al. 1993, Sierra et al. 2000), morfometría (Pérez 1996, Añez et al. 1997), morfología del atrio genital (Valenta et al. 1999), citogenética (Escobar 2000) y secuencias de genes mitocondriales y nucleares (Rojas et al. 2000).

El presente trabajo muestra los resultados del análisis electroforético de 16 loci isoenzimáticos con especial referencia en la determinación de los niveles de variabilidad genética, estructura y divergencia genética entre esas especies y a la determinación de la estructura espacial mediante autocorrelación en los procesos de especiación entre Lu. longiflocosa, Lu. torvida, Lu. spinicrassa, Lu. quasitownsendi y Lu. youngi, provenientes de focos endémicos colombianos de leishmaniasis cutánea.

\section{MATERIALES Y MÉTODOS}

Trabajo de Campo: Las capturas de flebótomos se realizaron en cinco localidades colombianas (Cuadro 1), por aspiración directa utilizando Trampas Shannon y cebo humano protegido. Con el fin de obtener una F1, las hembras recolectadas se colocaron en una jaula de muselina y se alimentaron con hámster anestesiado para asegurar la producción de huevos (Killick-Kendrick et al. 1997). Luego, se colocaron en vasos con yeso (hembras alimentadas y no alimentadas) para la ovoposición. Finalmente, se transportaron dentro de neveras de icopor al Insectario del Laboratorio de Entomología del INS (Bogotá - Cundinamarca). 
CUADRO 1

Sitios de colección de cinco especies colombianas de Lutzomyia (grupo verrucarum, serie townsendi)

y características geográficas y climáticas de los lugares de muestreo

TABLE 1

Sample localities of the five Colombian species of Lutzomyia (verrucarum group, serie townsendi) and geographic and climatic characteristics of the sampling places

Especie
Lu. longiflocosa
Lu. torvida
Lu. spinicrassa
Lu. quasitownsendi
Lu. youngi

Obtención de la F1 y Congelación: En el laboratorio las hembras se separaron individualmente en vasos de cría (Endris et al. 1982) con el fin de obtener machos y hembras descendientes de la misma madre. Los especimenes, machos y hembras de cada camada, se agruparon por sexo a medida que emergían y se preservaban congelados $\mathrm{a}-80^{\circ} \mathrm{C}$ en un congelador hasta su procesamiento para los isoenzimas. La madre y un descendiente macho de la primera generación, se aclararon en una solución de Hidróxido de Potasio al $10 \%$, se montaron en una mezcla de fenol-bálsamo de Canadá para su posterior identificación taxonómica siguiendo la clave de Young y Duncan (1994) y para conservarlos en la colección de referencia del INS, como material de referencia.

Método Electroforético: La electroforésis se realizó en geles verticales de poliacrilamida al $6 \%$. Se utilizaron dos soluciones tampón: TC (tris-citrato) y TBE (tris-borato-EDTA), para maximizar la separación electroforética de las isoenzimas y para visualizar las bandas en los geles se empleó un procedimiento de coloración histoquímico específico para cada uno de los 16 enzimas estudiados (Cuadro 2). Los tamaños muestrales fueron superiores a 30 individuos en todas las especies analizadas. Solo los ejemplares adultos (recién emergidos) fueron procesados para la electroforesis.

$\begin{array}{ccc}\text { Temp. }{ }^{\circ} \text { C. } & \begin{array}{c}\text { Elevación } \\ \text { (m.s.n.m.) }\end{array} & \text { Longitud y Latitud } \\ 25.0 & 1510 & 74^{\circ} 59^{\prime} 57^{\prime \prime}, 03^{\circ} 00^{\prime} 20^{\prime \prime} \\ 24.0 & 1425-1475 & 74^{\circ} 29^{\prime} 35^{\prime \prime}, 04^{\circ} 47^{\circ} 35^{\prime \prime} \\ 18.2 & 1690 & 73^{\circ} 30^{\prime} 36^{\prime \prime}, 05^{\circ} 01^{\prime} 27^{\prime \prime} \\ 21.6 & 1579 & 73^{\circ} 37^{\prime} 16^{\prime \prime}, 05^{\circ} 55^{\prime} 57^{\prime \prime} \\ 24.0 & 1150 & 76^{\circ} 12^{\prime} 00^{\prime \prime}, 04^{\circ} 05^{\prime} 12^{\prime \prime}\end{array}$

Análisis genético poblacional: Los alelos se identificaron alfabéticamente de acuerdo al valor estimado de frente de corrido (Rf). Los valores de $\mathrm{Rf}$ de las electroformas en el gel para cada locus se calcularon usando las referencias comerciales de las enzimas y está dado por la relación entre el valor de la distancia recorrida por una banda desde el origen del gel y el valor de la distancia recorrida por la banda de la enzima comercial respectiva. Los alelos codificados para la misma enzima se designaron numéricamente desde el más cercano al cátodo hasta el más cercano al ánodo. Se determinaron las frecuencias génicas de los respectivos marcadores, la heterocigosis esperada promedio, los estadísticos F de Wright, estimas indirectas de flujo génico y diversas distancias genéticas (Nei 1978, Cavalli-Sforza y Edwards 1967). Con las matrices de distancias genéticas se obtuvieron diversos dendrogramas aplicando los métodos UPGMA y Wagner (programa Biosys-2). Para ratificar las relaciones genéticas entre las especies estudiadas de Lutzomyia se aplicó un análisis de escalas multidimensionales con una función monótona (Kruskal 1964 a,b) a partir de las matrices de correlaciones y distancia Euclídea. Se utilizaron 50 iteraciones para calcular los estadísticos denominados stress $1 \mathrm{y}$ stress 2, los cuáles miden la bondad de ajuste de las distancias en el espacio configuracional con la función monótona de las distancias originales. 
CUADRO 2

Sistemas enzimáticos y número de loci codificados estudiados en cinco especies colombianas de Lutzomyia (grupo verrucarum, serie townsendi)

TABLE 2

Enzymatic systems and number of loci codified studied in five Colombian Lutzomyia species (verrucarum group, townsendi series)

\begin{tabular}{|c|c|c|c|c|c|}
\hline Enzima & Abrev. & $\mathrm{CE}^{\mathrm{a}}$ & \# Loci & Buffer $^{b}$ & $\mu 1 \operatorname{sln}^{c}$ \\
\hline \multicolumn{6}{|l|}{ Oxidoreductasas } \\
\hline Malato dehidrogenasa & Mdh & 1.1.1.37 & 2 & $\mathrm{TC}$ & 1 \\
\hline Enzima Malica & $\mathrm{Me}$ & 1.1 .1 .40 & 1 & TBE & 2 \\
\hline Isocitrato dehidrogenasa & Idh & 1.1.1.42 & 1 & $\mathrm{TC}$ & 1 \\
\hline 6-fosfogluconato dehidrogenasa & 6-pgdh & 1.1.1.44 & 1 & $\mathrm{TC}$ & 1 \\
\hline \multicolumn{6}{|l|}{ Transferasas } \\
\hline Aspartato aminotransferasa & Aat & 2.6.1.1 & 2 & $\mathrm{TC}$ & 2 \\
\hline Hexokinasa & $\mathrm{Hk}$ & 2.7.1.1 & 1 & TBE & 1 \\
\hline Arginina kinasa & Ark & 2.7.3.3 & 1 & $\mathrm{TC}$ & 1 \\
\hline Adenilato kinasa & Ak & 2.7 .4 .3 & 1 & $\mathrm{TC}$ & 1 \\
\hline Fosfoglucomutasa & Pgm & 2.7 .5 .1 & 1 & $\mathrm{TC}$ & 3 \\
\hline \multicolumn{6}{|l|}{ Hidrolasas } \\
\hline Esterasa & Est & 3.1.1.1 & 2 & TBE & 3 \\
\hline \multicolumn{6}{|l|}{ Liasas } \\
\hline Fumarato hidratasa & Fum & 4.2.1.2 & 1 & TBE & 2 \\
\hline Aconitato hidratasa & Aco & 4.2 .1 .3 & 1 & $\mathrm{TC}$ & 2 \\
\hline \multicolumn{6}{|l|}{ Isomerasas } \\
\hline Glucosa-6-fosfato isomerasa & Gpi & 5.3 .1 .9 & 1 & TBE & 0.5 \\
\hline Total & & & 16 & & \\
\hline
\end{tabular}

Abrev: $=$ Abreviatura del sistema enzimático estudiado. $\mathrm{CE}^{\mathrm{a}}=$ Enzyme Commission Classification System. Buffer ${ }^{\mathrm{b}}=\mathrm{TC}=$ tris-citrato; TBE = tris-borato-EDTA. $\mu 1 \operatorname{sln}^{\mathrm{c}}=$ Cantidad de sobrenadante de cada muestra (espécimen triturado) que se debe sembrar en el bolsillo del gel (Munstermann LE 1994).Abrev = abbreviators of the enzymatic system studied. CEa = Enzyme Commission Classification System. Bufferb $=\mathrm{TC}=$ tris-citrate; TBE $=$ tris-borate-EDTA. $\mu 1$ slnc $=$ Amount of each sample (specimen triturated) which should be analyzed in the gel (Munstermann LE 1994).

Una matriz gráfica ("minimum spanning tree") se superimpuso para observar la distorsión local probable generada por este proceso de reducción dimensional (Gower y Ross 1969).

Para llevar a cabo inferencias de cómo los procesos geográficos, o selectivos, han podido generar las especies estudiadas, se utilizó un análisis de autocorrelación espacial unidimensional mediante el índice I de Moran (Sokal 1986, Sokal y Oden 1978a,b, Sokal y Wartenberg 1983, Sokal et al. 1986, 1987, 1989). La autocorrelacion espacial es la dependencia del valor de una variable con respecto al valor de esa misma variable en poblaciones vecinas. Este tipo de análisis involucra cuatro pasos diferentes. Primero, se aplica un test de homogeneidad para comprobar cuál es el grado de diferenciación genética entre las especies estudiadas. Para ello se utilizó la probabilidad asociada al estadístico $\mathrm{F}_{\mathrm{ST}}$. Segundo, se procedió al cálculo del indice I de Moran. Este índice se calcula de la siguiente manera:

$$
\mathrm{I}=\mathrm{n} \sum_{\mathrm{ij}} \mathrm{w}_{\mathrm{ij}} \mathrm{z}_{\mathrm{i}} \mathrm{z}_{\mathrm{j}} / \mathrm{W} \sum_{\mathrm{i}=1 \ldots \mathrm{n}} \mathrm{z}_{\mathrm{i}}^{2}
$$

donde $\underline{n}$ es el número de localidades objeto de estudio, $\sum_{\mathrm{ij}}$ indica la suma sobre todas las especies $i$ de la primera a la $\mathrm{n}$ y $j$ de la primera a la $\mathrm{n}$, con $i$ diferente a $j ; \mathrm{w}_{\mathrm{ij}}$ es la ponderación dada a la relación entre las localidades $i$ y $j\left(\mathrm{w}_{\mathrm{ij}}\right.$ puede ser diferente de $\mathrm{w}_{\mathrm{ji}}$ ); $\mathrm{z}_{\mathrm{i}}=\mathrm{Y}_{\mathrm{i}}-\mathrm{Y}$, donde $\mathrm{Y}_{\mathrm{i}}$ es el valor de la variable $\mathrm{Y}$ para la especie 
$i$, e $\mathrm{Y}$ es la media de $\mathrm{Y}$ para todas las especies estudiadas; $\mathrm{y} \mathrm{W}=\sum_{\mathrm{ij}} \mathrm{w}_{\mathrm{ij}}$ es la suma de matriz de pesos, con $i$ diferente a $j$. Para muestras grandes, el valor de I oscila aproximadamente entre +1 a -1 y su valor aproximado esperado en ausencia de autocorrelación es 0 . Una figura con los coeficientes de autocorrelación frente a las distancias geográficas conforma un correlograma espacial. Los puntos geográficos fueron conectados mediante una red de Gabriel (Gabriel y Sokal 1969, Matula y Sokal 1980). Se realizaron dos análisis de autocorrelación espacial, uno con 3 clases de distancias (CD) cuyos límites superiores fueron 103, 176 y $302 \mathrm{~km}$ de distancia y otro con $4 \mathrm{CD}$, cuyos límites geográficos superiores fueron 76, 139, 176 y $302 \mathrm{~km}$ de distancia. En ambos análisis, el número de pares de comparaciones fue aproximadamente el mismo. La forma de los correlogramas permite determinar qué eventos evolutivos pudieron producir la aparición de las especies estudiadas. Estos análisis fueron aplicados a los 13 loci polimórficos y siempre se descarto el alelo con frecuencia genica mas baja en cada locus estudiado. Se determinó la significación estadística de los coeficientes de autocorrelación con el procedimiento de Bonferroni (Oden 1984). En tercer lugar se procedió a un análisis de correlación de las superficies de las variables mediante el coeficiente de correlación de Pearson ( $r$ ). Se contabilizó el porcentaje de correlaciones superior a $|0.7|$. Valores superiores a esta cifra muestran marcadores que poseen superficies similares. La última prueba realizada fue un análisis de similitud de los correlogramas. Para llevar a cabo esto, se calcularon las matrices de distancia de Manhattan entre todos los pares posibles de los 13 loci polimórficos. Este análisis es útil para investigar si cada una de las variables genéticas analizadas ha estado bajo la acción de los mismos procesos evolutivos espaciales. Sokal y Wartenberg (1983) y Sokal et al. (1989) mostraron con simulaciones computacionales que pares de correlogramas generados por los mismos procesos evolutivos tienen distancias de Manhattan promedio inferiores a 0.1 para el índice I de Moran. Para mostrar gráficamente las relaciones espaciales de las variables estudiadas se procedió a aplicar el algoritmo UPGMA y un procedimiento de clasificación no jerárquico, k-means (Spath 1983)

\section{RESULTADOS}

Variabilidad Genética: Tres (Ark, Aat-1 y Est-1) de los 16 loci enzimáticos fueron monomórficos en todas las especies (Cuadro 3). Dos loci codificaron para las enzimas Aat, Mdh y Est. El ámbito de los alelos encontrados por locus fue de 1 a 6 alelos. La mayoría de los loci polimórficos no mostraron más de dos o tres alelos, con la excepción de Aat-2 (4 alelos), Est-2 (5 alelos) y Gpi (6 alelos). Ocho de los loci analizados (Aco, 6-pgdh, Ak, Mdh-1, Mdh2, Fum, Hk y Me) presentaron las frecuencias génicas más elevadas para el mismo alelo en todas las especies estudiadas. Lu. longiflocosa, $L u$. torvida y $L u$. spinicrassa mostraron cuatro loci monomórficos, Lu. quasitownsendi tres y Lu. youngi 10 loci monomórficos. En diversas especies se presentaron alelos raros o privados "sensu" Slatkin (1985). Estas variantes pueden ser clasificadas como alelos de diagnóstico específico. Estos fueron los casos de Aat2-D, Gpi-A, Hk-C (Lu. longiflocosa), Gpi-F, Me-B (Lu. spinicrassa), Mdh-1-B, Mdh-2-C, Fum-A (Lu. quasitownsendi) e Idh-C, Est-2-A ( $L u$. youngi).

El porcentaje de loci polimórficos varió entre el 20 al $46.7 \%$. Lu. youngi mostró el menor valor y $\mathrm{Lu}$. quasitownsendi el mayor valor. El número promedio de alelos por locus varió de 1.3 (Lu. youngi) a 2.0 (Lu. torvida y Lu. quasitownsendi).

Los loci Gpi y Est-2 presentaron el mayor número de alelos y fueron también los loci con los más altos niveles de heterocigosis $(\mathrm{H})$. Para Gpi, el valor de H osciló entre 0.371 ( $L u$. quasitownsendi) a 0.689 (Lu. spinicrassa) y, para Est-2, H varió de 0.485 (Lu. longiflocosa) a 0.715 ( $\mathrm{Lu}$. torvida). Las isoenzimas que presentaron los niveles más bajos de $\mathrm{H}$, fueron $\mathrm{Ak}$ (Lu. longiflocosa $\mathrm{H}=0.018$ - Lu. spinicrassa $\mathrm{H}$ $=0.044)$ y 6-pgdh $(L u$. torvida $\mathrm{H}=0.020)$. Los valores de diversidad genética para cada especie 
CUADRO 3

Frecuencias alélicas para 13 loci polimórficos en cinco especies colombianas de Lutzomyia (grupo verrucarum, serie townsendi).

TABLE 3

Allele frequencies for 13 polymorphic loci in five Colombian Lutzomyia species (verrucarum group, townsendi series)

\begin{tabular}{|c|c|c|c|c|c|c|}
\hline \multicolumn{7}{|c|}{ Especie } \\
\hline Locus $^{\mathrm{a}}$ & Aloformas ${ }^{b}$ & longiflocosa & torvida & spinicrassa & quasitownsendi & youngi \\
\hline \multicolumn{7}{|c|}{ Aco } \\
\hline \multirow{4}{*}{$(\mathrm{N})$} & & 49 & 44 & 27 & 48 & 33 \\
\hline & A & .041 & 0 & .074 & .167 & 0 \\
\hline & B & .959 & .909 & .926 & .802 & 1.000 \\
\hline & $\mathrm{C}$ & 0 & .091 & 0 & .031 & 0 \\
\hline \multicolumn{7}{|c|}{ 6-pgdh } \\
\hline \multirow[t]{3}{*}{ (N) } & & 54 & 50 & 46 & 36 & 40 \\
\hline & $\mathrm{A}$ & 0 & .010 & 0 & 0 & 1.000 \\
\hline & B & 1.000 & .990 & 1.000 & 1.000 & 0 \\
\hline \multicolumn{7}{|c|}{$\mathrm{Ak}$} \\
\hline \multirow[t]{3}{*}{$(\mathrm{N})$} & & 55 & 50 & 45 & 41 & 42 \\
\hline & A & .009 & 0 & .022 & 0 & 0 \\
\hline & B & .991 & 1.000 & .978 & 1.000 & 1.000 \\
\hline \multicolumn{7}{|c|}{ Aat-2 } \\
\hline \multirow[t]{5}{*}{$(\mathrm{N})$} & & 45 & 39 & 46 & 45 & 33 \\
\hline & A & 0 & .526 & .065 & .056 & 0 \\
\hline & B & .978 & .385 & .859 & .833 & .803 \\
\hline & $\mathrm{C}$ & 0 & .090 & .076 & .111 & .197 \\
\hline & $\mathrm{D}$ & .022 & 0 & 0 & 0 & 0 \\
\hline \multicolumn{7}{|c|}{ Pgm } \\
\hline \multirow[t]{4}{*}{$(\mathrm{N})$} & & 48 & 37 & 29 & 47 & 37 \\
\hline & A & .354 & .486 & .448 & .809 & 0 \\
\hline & B & .625 & .500 & .448 & .117 & 1.000 \\
\hline & $\mathrm{C}$ & .021 & .014 & .103 & .074 & 0 \\
\hline \multicolumn{7}{|c|}{ Idh } \\
\hline \multirow[t]{4}{*}{$(\mathrm{N})$} & & 56 & 26 & 55 & 59 & 30 \\
\hline & A & .804 & .615 & .636 & .466 & .517 \\
\hline & B & .196 & .385 & .364 & .534 & .317 \\
\hline & $\mathrm{C}$ & 0 & 0 & 0 & 0 & .167 \\
\hline \multicolumn{7}{|c|}{ Mdh-1 } \\
\hline \multirow[t]{3}{*}{$(\mathrm{N})$} & & 38 & 40 & 45 & 45 & 30 \\
\hline & A & 1.000 & 1.000 & 1.000 & .989 & 1.000 \\
\hline & B & 0 & 0 & 0 & .011 & 0 \\
\hline \multicolumn{7}{|c|}{ Mdh-2 } \\
\hline \multirow[t]{4}{*}{$(\mathrm{N})$} & & 45 & 40 & 46 & 46 & 33 \\
\hline & A & 0 & .038 & 0 & .033 & 0 \\
\hline & B & 1.000 & .962 & 1.000 & .902 & 1.000 \\
\hline & $\mathrm{C}$ & 0 & 0 & 0 & .065 & 0 \\
\hline
\end{tabular}


CUADRO 3 (Continuación)

Frecuencias alélicas para 13 loci polimórficos en cinco especies colombianas de Lutzomyia (grupo verrucarum, serie townsendi).

TABLE 3 (Continued)

Allele frequencies for 13 polymorphic loci in five Colombian Lutzomyia species (verrucarum group, townsendi series)

\begin{tabular}{|c|c|c|c|c|c|c|}
\hline \multicolumn{7}{|c|}{ Especie } \\
\hline Locus $^{\mathrm{a}}$ & Aloformas ${ }^{b}$ & longiflocosa & torvida & spinicrassa & quasitownsendi & youngi \\
\hline \multicolumn{7}{|c|}{ Fum } \\
\hline \multirow[t]{4}{*}{$(\mathrm{N})$} & & 45 & 40 & 47 & 44 & 33 \\
\hline & $\mathrm{A}$ & 0 & 0 & 0 & .023 & 0 \\
\hline & B & .978 & .962 & 1.000 & .977 & 1.000 \\
\hline & $\mathrm{C}$ & .022 & .038 & 0 & 0 & 0 \\
\hline \multicolumn{7}{|c|}{ Gpi } \\
\hline \multirow[t]{7}{*}{$(\mathrm{N})$} & & 45 & 44 & 47 & 45 & 33 \\
\hline & A & .022 & 0 & 0 & 0 & 0 \\
\hline & B & .356 & .011 & 0 & .211 & 1.000 \\
\hline & $\mathrm{C}$ & .511 & .534 & .128 & .767 & 0 \\
\hline & $\mathrm{D}$ & .089 & .307 & .117 & 0 & 0 \\
\hline & $\mathrm{E}$ & .022 & .148 & .415 & .022 & 0 \\
\hline & $\mathrm{F}$ & 0 & 0 & .340 & 0 & 0 \\
\hline \multicolumn{7}{|c|}{$\mathrm{Hk}$} \\
\hline \multirow[t]{4}{*}{$(\mathrm{N})$} & & 51 & 45 & 52 & 50 & 39 \\
\hline & A & .951 & 1.000 & .962 & .990 & 1.000 \\
\hline & B & .039 & 0 & .038 & .010 & 0 \\
\hline & $\mathrm{C}$ & .010 & 0 & 0 & 0 & 0 \\
\hline \multicolumn{7}{|c|}{$\mathrm{Me}$} \\
\hline \multirow[t]{3}{*}{$(\mathrm{N})$} & & 45 & 40 & 47 & 46 & 33 \\
\hline & A & 1.000 & 1.000 & .979 & 1.000 & 1.000 \\
\hline & B & 0 & 0 & .021 & 0 & 0 \\
\hline \multicolumn{7}{|c|}{ Est-2 } \\
\hline \multirow[t]{6}{*}{$(\mathrm{N})$} & & 11 & 23 & 12 & 6 & 5 \\
\hline & A & 0 & 0 & 0 & 0 & .600 \\
\hline & B & 0 & .087 & 0 & .500 & .400 \\
\hline & $\mathrm{C}$ & 0 & .217 & .500 & .500 & 0 \\
\hline & $\mathrm{D}$ & .364 & .391 & .500 & 0 & 0 \\
\hline & $\mathrm{E}$ & .636 & .304 & 0 & 0 & 0 \\
\hline
\end{tabular}

(N) = Tamaño muestral. ${ }^{\mathrm{a}}=$ abreviaturas de las enzimas como en el cuadro 2 .

$(\mathrm{N})=$ Sample size. ${ }^{\mathrm{a}}=$ abbreviators of the enzymes like on the table 2 .

se muestran en cuadro 4. El promedio osciló entre 0.098 (Lu. youngi) a 0.215 (Lu. torvida). Por especies, los loci con valores de heterocigosis esperada más elevados para $\mathrm{Lu}$. longiflocosa fueron Idh, Est-2, Pgm y Gpi (0.319-0.610); para
Lu. torvida, Pgm, Aat-2, Gpi y Est-2 (0.5200.715); para Lu. spinicrassa, Idh, Est-2, Pgm, y Gpi (0.467-0.689); para Lu. quasitownsendi, Pgm, Gpi, Idh y Est-2 (0.331-0.545) y para $L u$. youngi, Aat-2, Est-2 e Idh (0.321-0.615). 
CUADRO 4

Variabilidad genética (número promedio de alelos por locus, porcentaje de loci polimórficos y heterocigosidad) en cinco especies colombianas de Lutzomyia (grupo verrucarum, serie townsendi) para 16 loci enzimáticos.

Entre paréntesis, la desviación estándar

TABLE 4

Genetic variability (average allele number per locus, percentage of polymorphic loci and heterozygosity) in five Colombian Lutzomyia species (verrucarum group, townsendi series) for 16 enzymatic loci.

In parentheses, the standard deviation

\begin{tabular}{|c|c|c|c|c|c|}
\hline \multirow[t]{2}{*}{ Especie } & \multirow[t]{2}{*}{$\begin{array}{c}\text { Tamaño promedio } \\
\text { de la muestra por locus }\end{array}$} & \multirow[t]{2}{*}{$\begin{array}{l}\text { No. promedio de } \\
\text { alelos por locus }\end{array}$} & \multirow[t]{2}{*}{$\begin{array}{c}\% \text { loci } \\
\text { polimórficos }^{\mathrm{a}}\end{array}$} & \multicolumn{2}{|c|}{$\begin{array}{l}\text { Promedio de } \\
\text { Heterocigosidad }\end{array}$} \\
\hline & & & & Observado & Esperado ${ }^{\mathrm{b}}$ \\
\hline Lu. longiflocosa & $\begin{array}{l}44.1 \\
(3.4)\end{array}$ & $\begin{array}{c}1.9 \\
(0.3)\end{array}$ & 26.7 & $\begin{array}{c}0.082 \\
(0.051)\end{array}$ & $\begin{array}{c}0.145 \\
(0.056)\end{array}$ \\
\hline Lu. torvida & $\begin{array}{l}40.5 \\
(2.0)\end{array}$ & $\begin{array}{c}2.0 \\
(0.3)\end{array}$ & 40.0 & $\begin{array}{c}0.086 \\
(0.047)\end{array}$ & $\begin{array}{l}0.215 \\
(0.071)\end{array}$ \\
\hline Lu. spinicrassa & $\begin{array}{l}40.4 \\
(3.2)\end{array}$ & $\begin{array}{c}1.9 \\
(0.2)\end{array}$ & 40.0 & $\begin{array}{c}0.099 \\
(0.044)\end{array}$ & $\begin{array}{c}0.189 \\
(0.065)\end{array}$ \\
\hline Lu. quasitownsendi & $\begin{array}{l}42.3 \\
(3.0)\end{array}$ & $\begin{array}{l}2.0 \\
(0.2)\end{array}$ & 46.7 & $\begin{array}{c}0.089 \\
(0.040)\end{array}$ & $\begin{array}{c}0.176 \\
(0.052)\end{array}$ \\
\hline Lu. youngi & $\begin{array}{l}31.7 \\
(2.7)\end{array}$ & $\begin{array}{c}1.3 \\
(0.2)\end{array}$ & 20.0 & $\begin{array}{c}0.048 \\
(0.042)\end{array}$ & $\begin{array}{c}0.098 \\
(0.054)\end{array}$ \\
\hline
\end{tabular}

Lu. youngi fue, de las cinco especies estudiadas, la que presentó las más bajas estimas en el número promedio de alelos por locus, en el porcentaje de loci polimórficos y en los promedios de heterocigosis observadas y esperadas.

Estructura Genética: Mediante la $\mathrm{f}$ de Robertson y Hill (1984), se determinaron las desviaciones de las proporciones genotípicas observadas respecto a las esperadas en equilibrio Hardy-Weinberg (EHW). Los porcentajes de desviación de este equilibrio para $L u$. longiflocosa, Lu. torvida y Lu. spinicrassa fueron de $69.23 \%$ (9 de los 13 loci polimórficos presentaron valores significativos a nivel $\mathrm{P}<0.05)$, para Lu. quasitownsendi $76.92 \%$ (10/13) y para Lu. youngi de $23.33 \%$ (3/13). Desviaciones significativas por exceso de homocigotos fueron detectadas para cuatro loci (Aco, Pgm, Fum y Est-2) en Lu. longiflocosa, seis loci (Aco, Aat-2, Pgm, Mdh-2, Fum, y Est-2) en Lu. torvida, seis loci (Ak,

Aat-2, Pgm, Gpi, Me y Est-2) en Lu. spinicrassa, cinco loci (Aco, Aat-2, Pgm, Fum y Est-2) en Lu. quasitownsendi y dos loci (Aat2, y Est-2) en Lu. youngi. Los marcadores que no estuvieron en EHW fueron diferentes entre las especies. Por ejemplo: Ak, Gpi y Me mostraron un exceso de homocigotos para $L u$. spinicrassa pero no para las demás especies. Mdh-2 presentó un exceso de homocigotos para $L u$. torvida, no encontrado en las otras cuatro especies.

La estructura genética para cada uno de los 13 loci polimórficos se analizó con los estadísticos F de Wright (1965) y se resume en el cuadro 5. El promedio de $\mathrm{F}_{\mathrm{IS}}$ (desviación respecto al EHW dentro de cada especie) para los 13 marcadores fue elevado y significativo $\left(\mathrm{F}_{\text {IS }}=0.5152, \mathrm{P}<0.00001\right)$. Los marcadores genéticos que contribuyeron más decisivamente a esta desviación significativa fueron $\mathrm{Me}$, Est-2, Fum, Pgm, Aco, Aat-2 y Ak, todos ellos por exceso de homocigotos. Otros marcadores 


\section{CUADRO 5}

Estimas de los estadísticos-F de Wright para los loci polimórficos analizados en cinco especies colombianas de Lutzomyia (grupo verrucarum, serie townsendi)

TABLE 5

Wright $F$-statistics for the polymorphic loci analyzed in five Colombian Lutzomyia (verrucarum group, serie townsendi) species

$\begin{array}{lccc}\text { Locus* } & \mathrm{F}_{\text {IS }} & \mathrm{F}_{\mathrm{ST}} & \mathrm{F}_{\text {IT }} \\ \text { Aco } & 0.7709 & 0.0631 & 0.7853 \\ \text { 6-pgdh } & -0.0101 & 0.9851 & 0.9850 \\ \text { Ak } & 0.6606 & 0.0115 & 0.6645 \\ \text { Aat-2 } & 0.7298 & 0.2224 & 0.7899 \\ \text { Pgm } & 0.7989 & 0.2942 & 0.8581 \\ \text { Idh } & -0.0101 & 0.0695 & 0.0601 \\ \text { Mdh-1 } & -0.0113 & 0.0086 & -0.0026 \\ \text { Mdh-2 } & 0.1074 & 0.0460 & 0.1484 \\ \text { Fum } & 0.8529 & 0.0158 & 0.8552 \\ \text { Gpi } & 0.0693 & 0.3416 & 0.3872 \\ \text { Hk } & -0.0384 & 0.0181 & -0.0196 \\ \text { Me } & 1.0000 & 0.0166 & 1.000 \\ \text { Est-2 } & 1.0000 & 0.2383 & 1.000 \\ \text { Promedio } & 0.5152 & 0.2884 & 0.6550\end{array}$

mostraron valores $\mathrm{F}_{\mathrm{IS}}$ negativos, aunque no significativos debido a exceso de heterocigotos (6-pgdh, Idh, Mdh-1 y Hk). El valor promedio de $\mathrm{F}_{\mathrm{IT}}$ (desviación respecto al EHW para el conjunto global de todas las especies de Lutzomyia analizadas) fue significativamente elevado $\left(\mathrm{F}_{\mathrm{IT}}=0.655 ; \mathrm{P}<0.00001\right)$. Ocho marcadores presentaron una diferenciación significativa para $\mathrm{F}_{\mathrm{IT}}$, por un conspicuo exceso de homocigotos, Me, Est-2, 6-pgdh, Pgm, Fum, Aat-2, Aco, y Ak. Mdh-1 y Hk mostraron valores de $\mathrm{F}_{\mathrm{IT}}$ negativos aunque no significativos. Globalmente, el grado de heterogeneidad inter - especies es medido mediante el estadístico $\mathrm{F}_{\mathrm{ST}}(=0.2884, \mathrm{P}<0.00002)$. Este valor es elevado y muestra una pronunciada diferenciación genética entre las especies, ratificando que son unidades reproductivas aisladas. Los marcadores que mostraron heterogeneidad más elevada fueron 6-pgdh, Gpi, Pgm, Est-2 y Aat2. Por lo tanto, esos son los marcadores que más discriminan entre los taxones estudiados.

\section{CUADRO 6}

Identidad genética (I) y distancia genética (D) de Nei (1978) entre cinco especies colombianas de Lutzomyia (grupo verrucarum, serie townsendi)

TABLE 6

Genetic identity (I) and genetic distance (D) (Nei 1978) between five Colombian Lutzomyia species (verrucarum group, townsendi series)

$\begin{array}{lccccc}\text { Especie } & 1 & 2 & 3 & 4 & 5 \\ \text { (1) Lu. longiflocosa } & * * * * * & .041 & .051 & .075 & .169 \\ \text { (2) Lu. torvida } & .959 & * * * * * & .041 & .058 & .217 \\ \text { (3) Lu. spinicrassa } & .950 & .960 & * * * * * & .062 & .206 \\ \text { (4) Lu. quasitownsendi } & .928 & .944 & .940 & * * * * * & .228 \\ \text { (5) Lu. youngi } & .845 & .805 & .814 & .796 & * * * * *\end{array}$


Basándose en el promedio de $\mathrm{F}_{\mathrm{ST}}$, se calculó una estima teórica de flujo génico, $\mathrm{Nm}$ (número medio de inmigrantes que entran en una población por generación) $=0.6168$, lo cual revela que, efectivamente, las poblaciones analizadas no intercambian individuos entre sí. Incluso, si se aplica un modelo isla n-dimensional, la estima de flujo génico es prácticamente nula $(\mathrm{Nm}=0.2435)$

Diferenciación Genética: Los valores de la identidad (I) y de la distancia genética (D) de Nei (1978) entre las especies estudiadas se muestran en el cuadro 6. La divergencia genética de $L u$. youngi respecto a las otras especies colombianas fue considerable, con valores de D oscilando entre 0.169-0.228. Las otras cuatro especies presentaron valores menores, oscilando entre 0.041 y 0.075 ). Las especies más relacionadas fueron $L u$. longiflocosa-Lu. torvida y Lu torvida-Lu. spinicrassa mientras que las genéticamente más distantes fueron $L u$. torvida-Lu. youngi y Lu. quasitownsendi-Lu. youngi (0.796). La relación genética entre las 5 especies se presenta gráficamente en el dendrograma con el método de Wagner (Fig .1). El análisis de escalas multidimensionales (MSD), tanto con la matriz de correlaciones como con la matriz de distancias euclídea, mostró que las especies $L u$. longiflocosa, Lu. spnicrassa y Lu. torvida están altamente relacionadas entre sí, mientras que las especies $L u$. quasitownsendi y, especialmente, Lu. youngi son mucho más distantes. Lu. quasitownsendi estaría relacionada con $L u$. torvida y $L u$. youngi con $L u$. longiflocosa (Fig. 2). El ajuste de los datos en MSD fue extremadamente bueno ya que los estadísticos stress 1 y 2 fueron igual a 0 , en todos los casos.

Autocorrelación Espacial: Se realizaron diferentes análisis de autocorrelación espacial. En primera instancia, se tomaron tres clases de distancia (CD) cuyos límites geográficos superiores fueron 103, 176 y $302 \mathrm{Km}$. También se realizó un análisis con 4 CD cuyos límites geográficos superiores fueron 76, 139, 176 y $302 \mathrm{Km}$. Para el caso de tres CD, el $11.49 \%$ de los índices I de Moran fue significativo (10 de 87 casos). Globalmente, únicamente el alelo Gpi-B mostró un correlograma globalmente significativo $(\mathrm{p}=0.029)$ con la tercera $\mathrm{CD}$ $(176-302 \mathrm{~km})$ significativamente negativa. Es decir, en este caso las especies más distantes geográficamente fueron también las que difieren más fuertemente para este marcador. 6-pgdh-B, Pgm-B y Est2-C también presentaron la primera $\mathrm{CD}$ positiva (relación fuerte

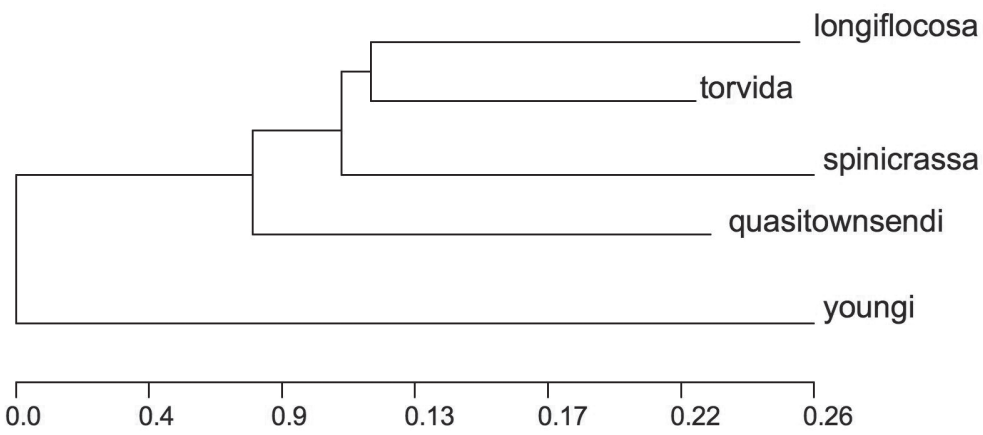

Fig 1. Dendrograma generado por el método Wagner con la distancia genética de Nei (1978) aplicado a cinco especies colombianas de Lutzomyia (grupo verrucarum, serie townsendi). Longitud total del árbol = 0.927; f de Farris =0.014; F de Prager y Wilson $=3.819$; Porcentaje de desviación estándar $=4.798$; Coeficiente de correlación cofenético $=0.986$.

Fig 1. Dendrogram generated by the Wagner algorithm with the Nei's (1978) genetic distance applied to five Colombian Lutzomyia species (verrucarum group, townsendi series). Total length tree $=0.927$; Farris's $\mathrm{f}=0.014$; Prager and Wilson's $\mathrm{F}=3.819 ;$ Percentage of standard deviation $=4.798$; Cophenetic correlation coefficient $=0.986$. 

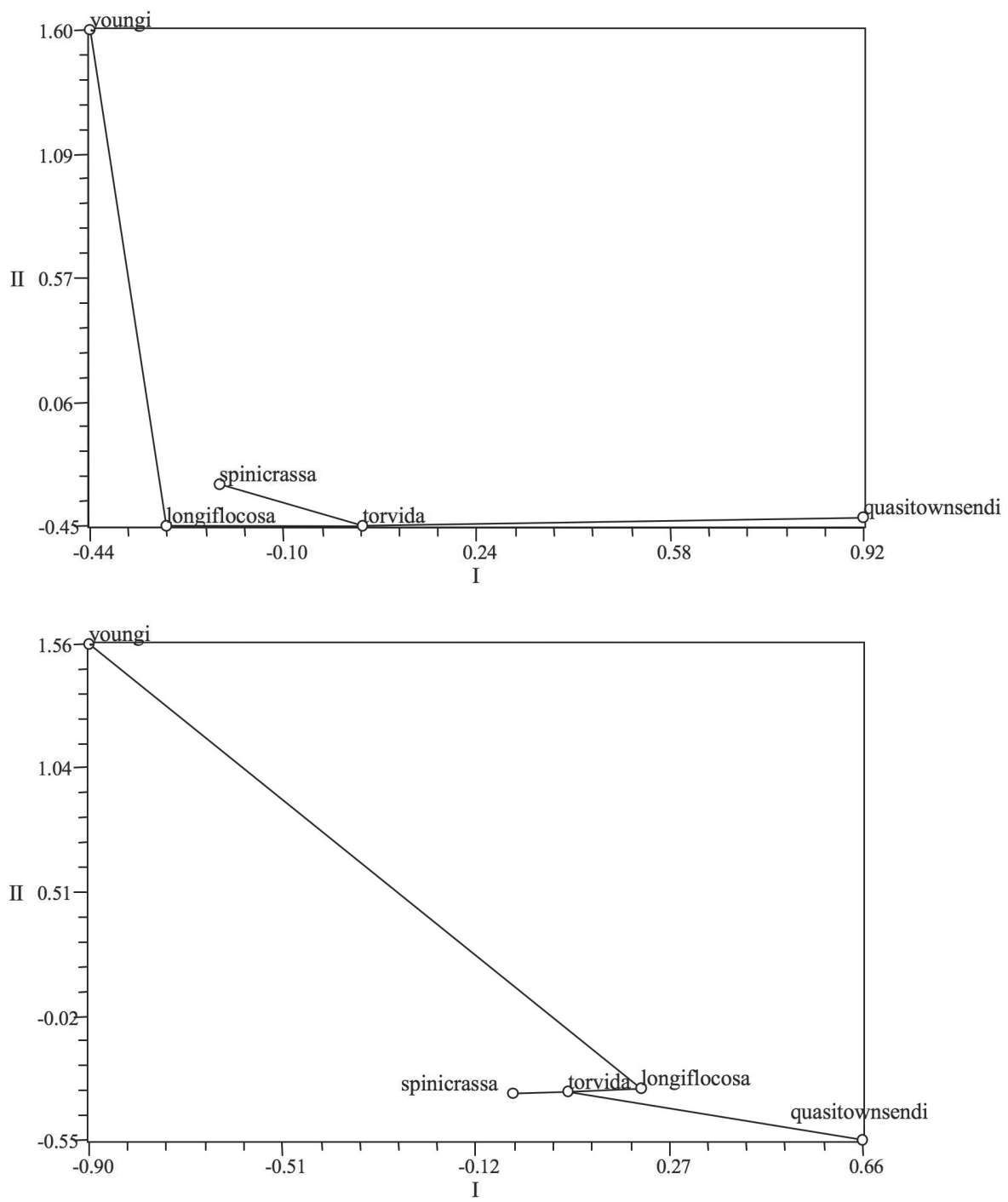

Fig 2. Análisis de Escalas multidimensionales (MSD) aplicado a cinco especies colombianas de Lutzomyia (grupo verrucarum, serie townsendi) a partir de la matriz de correlaciones (A) y a partir de la matriz de distancia Euclidea (B). Un árbol mínimo expandido se ha superimpuesto para determinar la relación entre las especies después de proceder a una reducción de las dimensiones.

Fig 2. Multidimensional Scale Analysis (MSA) applied to five Colombian Lutzomyia species ((verrucarum group, townsendi series) throughout a correlation matrix (A) and throughout a Euclidean distance matrix (B). A minimum spanning tree was superimposed to determine the relationships between the species after to reduce spatial dimensions. 
entre especies próximas geográficamente) y la última $\mathrm{CD}$ significativamente negativa (diferencias considerables entre especies distantes geográficamente). Sin embargo, para ninguno de estos 3 alelos, el correlograma global fue significativo. Aat2-C, Idh-A y Est2-E exhiben correlogramas con aspecto de "depresión" o clina circular, ya que de 103 a $176 \mathrm{~km}$ existe una fuerte caída en la similitud genética. Sin embargo, globalmente, estos correlogramas no fueron significativos. Esto es, solo se puede afirmar que existe una tendencia a la presencia de una clina circular o, en un hábitat casi lineal, la existencia de dos clinas convergentes cerca del centro de la zona estudiada pero sin alcanzar una significación estadística. Gpi-E, Hk-A y Hk-B mostraron un valor negativo para la primera $\mathrm{CD}$ (diferencias genéticas remarcables entre especies cercanas geográficamente). Sin embargo, para el primer alelo se obtuvo en la 2 CD (103-176 km) un valor significativamente positivo, indicando que las especies a esta distancia son más similares genéticamente entre sí de lo esperado simplemente por azar. Para los alelos HK-A y HK-B, las especies más distantes geográficamente fueron también las significativamente más similares. No obstante, ninguno de esos correlogramas fue significativo globalmente. En el análisis de $4 \mathrm{CD}$, solo el $9.82 \%$ de los coeficientes fue significativo $(11 / 112)$, valor que no es significativamente diferente del error tipo I del $5 \%$. Al igual que en el caso anterior, Gpi-B fue el único alelo que presentó un correlograma globalmente significativo $(\mathrm{p}=0.023)$. Otros alelos que presentaron algunos coeficientes significativos, pero sin que los correlogramas globales alcanzaran ese estado, fueron: 1/ 6-pgdh-B, con la $1 \mathrm{CD}$ positiva y significativa (fuerte parecido entre las especies situadas a menos de $76 \mathrm{~km}$ de distancia) y la última CD fuertemente negativa (176$302 \mathrm{~km}$ ), indicando fuerte disimilitud entre las especies situadas a esa distancia. 2/ Para Pgm-A y Pgm-B, también se dio una fuerte disimilitud genética entre las especies situadas más distantemente. 3/ Aat2-B mostró una fuerte disimilitud genética entre las especies más cercanas geográficamente. 4/ Idh-B, Est2-C y
Est2-E presentaron aspecto de clina circular ya que la $3 \mathrm{CD}(139-176 \mathrm{~km})$ fue significativamente negativa. Finalmente, Fum-c mostró la $1 \mathrm{CD}$ significativamente positiva, indicando un fuerte parecido entre las especies separadas en los primeros $76 \mathrm{~km}$ de distancia. Las restantes variables no mostraron ningún tipo de sistema especial en ninguno de los análisis. Esto indica que, en general, la distancia geográfica entre las especies estudiadas no es un factor demasiado trascendente en los fenómenos de especiación entre ellas. El correlograma promedio para las 28 variables estudiadas no fue significativo, ratificando la idea anterior (Cuadro 7).

El porcentaje de correlaciones con un valor superior a $|0.7|$ fue del $18.41 \%$, lo cual fue superior significativamente al error del $5 \%$ $\left(151 / 820 ; \chi^{2}=71.377,1\right.$ grado de libertad, $\mathrm{P}$ $<0.000001)$. Eso significa que una proporción significativa de las superficies de un conjunto de variables estudiadas están correlacionadas significativamente.

Por el contrario, el porcentaje de distancias de Manhattan entre pares de correlogramas inferiores a 0.1 para el análisis de autocorrelación espacial con $3 \mathrm{CD}$ fue extremadamente bajo $(3.44 \%, 13 / 378)$. Este valor no se diferenció del error tipo del $5 \%$ y, por lo tanto, se concluye que, desde el punto de vista espacial, no existen fuerzas evolutivas globales que hayan afectado de forma similar a las variables analizadas. El análisis UPGMA (Fig. 3) muestra algunas asociaciones espacialmente importantes como serían los casos de Gpi-c, Gpi-F y Me-A; Pgm-A y Pgm-B; y, finalmente, Hk-A y Hk-B. Sin embargo, globalmente ese número de asociaciones es muy bajo. Exactamente las mismas conclusiones se alcanzan con el análisis de autocorrelación espacial con 4 CD. El porcentaje de pares de correlogramas con distancias de Manhattan inferiores a 0.1 fue tan solo de $3.17 \%$ (12/378), lo cual ni siquiera alcanza el error tipo del $5 \%$. El correspondiente análisis UPGMA mostró que solo las variables Mdh1-A, Mdh2-c, Gpi-F y Me-A; los alelos Mdh2-B y Gpi-E; Hk-A y Hk-B; y Pgm-A y Pgm-B, presentaron pequeños valores de las distancias de Manhattan, lo cual es compatible 
CUADRO 7

Coeficientes de autocorrelación espacial con el índice I de Moran de los alelos independientes de los 13 loci polimórficos analizados para cinco especies colombianas de Lutzomyia (grupo verrucarum, serie townsendi)

TABLE 7

Spatial autocorrelation coefficients, by using the Moran's I index, of the independent alleles at the 13 polymorphic loci analyzed for five Colombian Lutzomyia species (verrucarum group, townsendi series)

\begin{tabular}{|c|c|c|c|c|c|}
\hline \multicolumn{6}{|c|}{ (A) } \\
\hline Alelos & $3 \mathrm{CD}$ & $103 \mathrm{Km}$ & $176 \mathrm{Km}$ & $302 \mathrm{Km}$ & PG \\
\hline Aco-A & & -0.30 & -0.16 & -0.28 & $\mathrm{P}=1.000$ \\
\hline Aco-B & & 0.00 & -0.12 & -0.54 & $\mathrm{P}=0.533$ \\
\hline 6-pgdh-B & & 0.25 & -0.17 & $-0.68 *$ & $\mathrm{P}=0.125$ \\
\hline Ak-B & & -0.34 & -0.59 & 0.08 & $\mathrm{P}=0.442$ \\
\hline Aat2-A & & -0.63 & 0.01 & -0.16 & $P=0.319$ \\
\hline Aat2-B & & -0.80 & -0.12 & 0.06 & $P=0.168$ \\
\hline Aat2-C & & 0.01 & $-0.92 *$ & 0.06 & $\mathrm{P}=0.150$ \\
\hline Pgm-A & & 0.16 & 0.02 & -0.76 & $P=0.157$ \\
\hline Pgm-B & & 0.21 & 0.03 & $-0.80 *$ & $\mathrm{P}=0.125$ \\
\hline Idh-A & & -0.09 & $-1.12^{*}$ & 0.28 & $\mathrm{P}=0.081$ \\
\hline Idh-B & & 0.03 & -0.60 & -0.20 & $P=0.602$ \\
\hline Mdh1-A & & -0.58 & -0.17 & -0.06 & $\mathrm{P}=0.428$ \\
\hline Mdh2-B & & -0.33 & -0.34 & -0.13 & $\mathrm{P}=1.000$ \\
\hline Mdh2-C & & -0.58 & -0.17 & -0.06 & $\mathrm{P}=0.428$ \\
\hline Fum-B & & 0.23 & -0.63 & -0.32 & $\mathrm{P}=0.443$ \\
\hline Fum-C & & 0.13 & -0.76 & -0.15 & $P=0.465$ \\
\hline Gpi-B & & 0.13 & 0.30 & $-0.94 * *$ & $\mathrm{P}=0.029 *$ \\
\hline Gpi-C & & -0.11 & -0.16 & -0.42 & $\mathrm{P}=0.955$ \\
\hline Gpi-D & & -0.67 & 0.15 & -0.24 & $\mathrm{P}=0.422$ \\
\hline Gpi-E & & -0.47 & $0.41^{*}$ & -0.58 & $\mathrm{P}=0.087$ \\
\hline Gpi-F & & -0.17 & -0.17 & -0.38 & $\mathrm{P}=0.926$ \\
\hline Hk-A & & -0.46 & -0.98 & $0.45^{*}$ & $\mathrm{P}=0.090$ \\
\hline Hk-B & & -0.43 & -0.96 & $0.42 *$ & $\mathrm{P}=0.123$ \\
\hline Me-A & & -0.17 & -0.17 & -0.38 & $P=0.926$ \\
\hline Est2-B & & -0.53 & -0.58 & 0.21 & $\mathrm{P}=0.330$ \\
\hline Est2-C & & 0.44 & -0.07 & $-0.90 *$ & $\mathrm{P}=0.132$ \\
\hline Est2-D & & -0.62 & 0.17 & -0.04 & $P=0.656$ \\
\hline Est2-E & & 0.34 & $-0.99 *$ & -0.14 & $\mathrm{P}=0.147$ \\
\hline Promedio & & -0.19 & -0.33 & -0.23 & \\
\hline
\end{tabular}


CUADRO 7 (Continuación)

Coeficientes de autocorrelación espacial con el índice I de Moran de los alelos independientes de los 13 loci polimórficos analizados para cinco especies colombianas de Lutzomyia (grupo verrucarum, serie townsendi)

TABLE 7(Continued)

Spatial autocorrelation coefficients, by using the Moran's I index, of the independent alleles at the 13 polymorphic loci analyzed for five Colombian Lutzomyia species (verrucarum group, townsendi series)

\begin{tabular}{|c|c|c|c|c|c|c|}
\hline \multicolumn{7}{|c|}{ (B) } \\
\hline Alelos & $4 \mathrm{CD}$ & $76 \mathrm{Km}$ & $103 \mathrm{Km}$ & $176 \mathrm{Km}$ & $302 \mathrm{Km}$ & PG \\
\hline Aco-A & & 0.37 & -0.55 & -0.26 & -0.35 & $\mathrm{P}=0.511$ \\
\hline Aco-B & & -0.14 & 0.33 & -0.50 & -0.74 & $\mathrm{P}=0.293$ \\
\hline 6-pgdh-B & & $0.25 *$ & -0.18 & 0.26 & $-1.00 *$ & $\mathrm{P}=0.091$ \\
\hline Ak-B & & -0.77 & -0.34 & 0.18 & -0.09 & $\mathrm{P}=0.635$ \\
\hline Aat2-A & & -0.58 & -0.32 & 0.22 & -0.03 & $\mathrm{P}=0.513$ \\
\hline Aat2-B & & $-0.91 *$ & -0.42 & 0.38 & -0.06 & $\mathrm{P}=0.178$ \\
\hline Aat2-C & & 0.02 & -0.80 & 0.03 & -0.06 & $\mathrm{P}=0.354$ \\
\hline Pgm-A & & 0.05 & 0.27 & -0.20 & $-1.00 *$ & $\mathrm{P}=0.091$ \\
\hline Pgm-B & & 0.21 & 0.24 & -0.27 & $-1.04 *$ & $\mathrm{P}=0.072$ \\
\hline Idh-A & & -0.09 & -0.46 & -0.82 & 0.24 & $\mathrm{P}=0.374$ \\
\hline Idh-B & & -0.14 & 0.32 & $-1.23 *$ & -0.24 & $\mathrm{P}=0.108$ \\
\hline Mdh1-A & & -0.38 & -0.17 & -0.38 & -0.17 & $\mathrm{P}=1.000$ \\
\hline Mdh2-B & & -0.76 & 0.27 & -0.40 & -0.34 & $\mathrm{P}=0.348$ \\
\hline Mdh2-C & & -0.38 & -0.17 & -0.38 & -0.17 & $\mathrm{P}=1.000$ \\
\hline Fum-B & & 0.02 & -0.48 & -0.13 & -0.29 & $\mathrm{P}=1.000$ \\
\hline Fum-C & & $0.84 *$ & -1.03 & -0.50 & -0.03 & $\mathrm{P}=0.189$ \\
\hline Gpi-B & & 0.08 & $0.39 *$ & -0.06 & $-1.23 *$ & $\mathrm{P}=0.023^{*}$ \\
\hline Gpi-C & & -0.51 & -0.13 & 0.09 & -0.43 & $\mathrm{P}=1.000$ \\
\hline Gpi-D & & -0.17 & -0.44 & 0.05 & -0.31 & $\mathrm{P}=1.000$ \\
\hline Gpi-E & & -0.66 & 0.24 & -0.40 & -0.37 & $\mathrm{P}=0.322$ \\
\hline Gpi-F & & -0.38 & -0.17 & -0.38 & -0.17 & $\mathrm{P}=1.000$ \\
\hline Hk-A & & -0.91 & -0.61 & 0.33 & 0.16 & $\mathrm{P}=0.453$ \\
\hline Hk-B & & -0.85 & -0.65 & 0.46 & 0.08 & $\mathrm{P}=0.396$ \\
\hline Me-A & & -0.38 & -0.17 & -0.38 & -0.17 & $\mathrm{P}=1.000$ \\
\hline Est2-B & & -0.43 & -0.39 & -0.23 & -0.01 & $\mathrm{P}=0.945$ \\
\hline Est2-C & & 0.72 & 0.30 & $-1.24 *$ & -0.79 & $\mathrm{P}=0.141$ \\
\hline Est2-D & & -0.53 & -0.22 & -0.00 & -0.26 & $\mathrm{P}=1.000$ \\
\hline Est2-E & & 0.68 & -0.67 & $-1.32 *$ & 0.25 & $\mathrm{P}=0.077$ \\
\hline Promedio & & -0.20 & -0.21 & -0.25 & -0.32 & \\
\hline
\end{tabular}

Se analizaron tres (A) y cuatro (B) clases de distancias (CD), respectivamente. $\mathrm{PG}=$ Probabilidad global del correlograma. Coeficiente de autocorrelación individual significativo $*=\mathrm{P}<0.05 ; * *=\mathrm{P}<0.01$.

Three (A) and four (B) distance classes (CD) were analyzed, respectively. $\mathrm{PG}=$ Overall correlogram probability. Individual significant autocorrelation coefficient $*=\mathrm{P}<0.05$ : * $=\mathrm{P}<0.01$. 

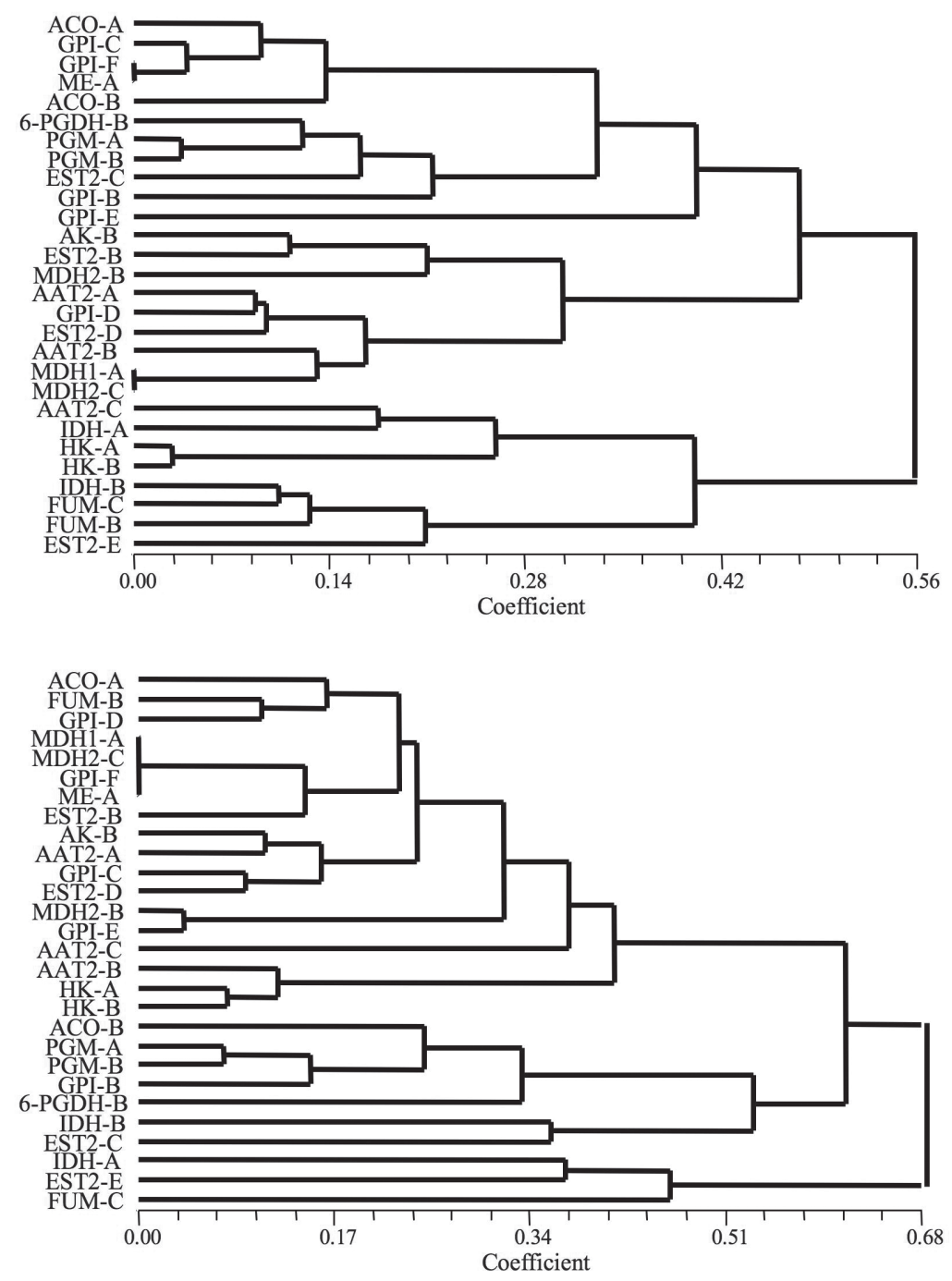

Fig 3. Dendrogramas generados con el algoritmo UPGMA a partir de las matrices de Manhattan entre pares de correlogramas de cada uno de los alelos analizados mediante autocorrelación espacial con el índice I de Moran. (A) Habiendo definido 3 clases de Distancia, (B) habiendo definido 4 clases de distancias. Los alelos más cercanos reflejados en esos árboles son lo que muestran haber sido influenciados por los mismos eventos evolutivos espaciales a lo largo de su historia.

Fig 3. Dendrograms generated with the UPGMA algorithm throughout the Manhattan matrices among correlogram pairs for each one of the alleles analyzed by employing spatial autocorrelation with the Moran's I index. (A) Defining 3 distance classes, (B) defining 4 distance classes. The most neighbor alleles reflected in these trees were those showing an influence of the same spatial evolutionary events generating their spatial patterns through their histories. 
con el hecho que los mismos eventos evolutivos espaciales hayan esculpido de igual forma sus correspondientes correlogramas. Los restantes marcadores poseen correlogramas con formas propias no similares entre sí. Se puede concluir globalmente que la dinámica espacial de las variables analizadas está afectada por muy diferentes eventos evolutivos.

\section{DISCUSIÓN}

Diversidad genética: El polimorfismo isoenzimático en las cinco especies estudiadas de Lutzomyia, (grupo verrucarum, serie townsendi) fue elevado. Trece de los 16 marcadores analizados fueron polimórficos $(81,3 \%)$. El porcentaje de polimorfismo encontrado es lo suficientemente elevado como para utilizar esos marcadores con fines genético poblacionales e, incluso, filogenéticos. Kreutzer et al. (1990) encontraron un porcentaje similar de marcadores polimórficos en este grupo, aunque ligeramente más elevado (95.2 \%).

Los dos loci con el mayor número de alelos fueron Gpi (6 alelos) y Est-2 (5 alelos), lo cual, puede indicar que ambos son los que exhiben una mayor acción de la selección natural diversificante entre las especies estudiadas y/o sus tasas de mutación por generación son más elevadas.

Cuatro de las cinco especies presentaron alelos diagnósticos, es decir, restringidos a una sola especie. De este modo, Aat-2-D, Gpi-A y $\mathrm{Hk}-\mathrm{C}$ son útiles para diferenciar a $\mathrm{Lu}$. longiflocosa, Gpi-F y Me-B a Lu. spinicrassa, Mdh1-B, Mdh2-C y Fum-A a Lu. quasitownsendi y Est-2-A e Idh-C a Lu. youngi. Esto muestra la utilidad de los marcadores isoenzimáticos en su poder de discriminación de especies en el seno del género Lutzomyia.

El nivel medio de heterocigosis esperada para las cinco especies no fue excesivamente elevado (0.098-0.215). Esto puede ser atribuido a la presencia reciente de efectos fundadores recientes en las localidades muestreadas, o a un bajo flujo genético entre poblaciones en el seno de cada especie, con incidencia de la deriva genética en cada una de ellas. Sin embargo, los marcadores isoenzimáticos tradicionalmente muestran valores de diversidad genética ostensiblemente más bajos que otro tipo de marcadores moleculares, como microsatélites, VNTRs o AFLPs. Es frecuente que áreas recientemente colonizadas posean poblaciones con menor diversidad genética respecto a poblaciones originales o ancestrales. Generalmente, las áreas de dispersión original de una especie son las que presentan niveles más elevados de variabilidad genética (Dobzhansky 1971). El valor de H para Lu. youngi fue 0.098, el valor menor, mientras que para $L u$. torvida fue 0.215 (el valor mayor), lo cual indicaría que, de las cinco especies analizadas, Lu. youngi poseería la menor distribución geográfica y sería la población más moderna formada a partir de un cuello de botella reciente, mientras que $L u$. torvida sería la especie con mayor distribución geográfica original y la más antigua, si se tiene en consideración los niveles de diversidad genética. Sin embargo, la distribución geográfica actual para estas dos especies es contraria a lo esperado por los resultados genéticos, ya que $L u$. youngi presenta una distribución en Centroamérica (Costa Rica, dos localidades) y Suramérica (Venezuela, 6 localidades - Colombia, 8 localidades), mientras que Lu. torvida posee una distribución geográfica restringida a Colombia, en una sola localidad. Kreutzer et al. (1990) encontraron un ámbito de $\mathrm{H}=0.082-0.171$, para 10 especies de Lutzomyia del grupo verrucarum. Estos valores están dentro de un ámbito muy similar al reportado en el presente estudio.

Estructura genética: Se detectaron fuertes desviaciones respecto al EHW, predominando un exceso de homocigotos para el global de los loci analizados para las cinco especies estudiadas. Lu. quasitownsendi presentó el mayor número de marcadores genéticos en desequilibrio HW (76.92\%), siendo así, la especie que más se apartó del citado equilibrio. Las desviaciones afectaron principalmente a cinco loci, Aco, Aat-2, Pgm, Fum y Est-2. Este hecho puede estar favorecido por diversas causas: (1) Es probable que se esté dando efecto Wahlund 
en el seno de cada una de las citadas especies debido a subdivisión geográfica real y/o a subdivisión temporal generacional en el seno de cada poblaciones. Se encontraron algunos marcadores, como Idh y Hk, que generalmente estuvieron en EHW en todas las especies estudiadas, mientras que otros mostraron un claro exceso de homocigotos en todas las especies (Pgm, Fum y Est-2). Esto no es compatible con un exceso de homocigotos por endogamia, porque ésta debe actuar de manera idéntica en todo el genoma y el exceso de homocigotos debería, por lo tanto, ser general para todos los marcadores analizados. El hecho que algunos marcadores presenten exceso de homocigotos y otros estén en equilibrio o presenten exceso de heterocigotos, parece favorecer la explicación de un exceso de homocigotos por efecto Wahlund (= subdivisión) más que por endogamia como posible explicación evolutiva de los resultados encontrados. (2) La sistematicidad con la que ciertos marcadores presentan exceso de homocigotos en todas las especies analizadas, también, puede ser un síntoma relacionado con la actuación de fuerzas selectivas positivas para las combinaciones homocigóticas en esos marcadores. (3) Sin embargo, no se puede descartar la existencia de alelos nulos, al menos, en algunos de los marcadores isoenzimáticos utilizados que mostraron exceso de homocigotos. (4) Otra posibilidad es que el tipo de muestreo y selección de los individuos analizados no se haya realizado realmente al azar. Sin embargo, eso no explicaría porqué sistemáticamente ciertos marcadores no están en equilibrio y otros sí en todas las especies.

$\mathrm{El}$ análisis jerarquizado con las $\mathrm{F}$ de Wright mostró valores elevados y significativos para cada uno de los estadísticos empleados. El valor promedio de $\mathrm{F}_{\mathrm{IT}}$ fue positivo y significativo $\left(\mathrm{F}_{\mathrm{IT}}=0.655\right)$ con un conspicuo exceso de homocigotos a nivel global en todas las especies. Este resultado revela, efectivamente, que las cinco poblaciones estudiadas de Lutzomyia representan especies reales, porque el intercambio genético entre ellas es extremadamente bajo. El estadístico promedio $\mathrm{F}_{\mathrm{IS}}$ fue también significativamente positivo (0.515), con exceso de homocigotos en el interior de cada especie. Esto ratifica que en el seno de cada una de ellas también existe una fuerte división genética. La heterogeneidad entre las cinco especies fue altamente significativa $\left(\mathrm{F}_{\mathrm{ST}}=0.288\right)$, mostrando que, en promedio, cada especie únicamente posee el $71.2 \%$ de la diversidad genética total encontrada en Lutzomyia. El locus 6-pgdh mostró el valor de $\mathrm{F}_{\mathrm{ST}}$ más elevado, seguido por Gpi, Pgm y Est-2. Estos son, pues, los marcadores que más fuertemente discriminan entre las especies analizadas. Los loci que muestran niveles inusualmente elevados de subdivisión poblacional respecto a otros loci, se señalan frecuentemente como indicadores de selección positiva diversificante por adaptación local. Este puede ser el caso para esos marcadores. Contrariamente, el locus Mdh-1 presentó el valor más pequeño de $\mathrm{F}_{\mathrm{ST}}$. Para este locus no se pueden descartar fenómenos selectivos constrictivos que lo estén afectando.

Las estimas de flujo génico $(\mathrm{Nm}=0.62$ y $\mathrm{Nm}=0.24)$, indican una virtual ausencia de intercambio genético entre las poblaciones analizadas, lo cual refuerza el concepto que realmente cada una de ellas representa un linaje evolutivamente independiente. Wright (1931, 1943) mostró que si $\mathrm{Nm}>1$, el flujo génico es suficientemente elevado para anular las diferencias genéticas entre las poblaciones involucradas que estén en equilibrio respecto a la deriva genética y al flujo génico en un modelo neutral isla infinito. En un modelo "stepping-stone", Nm debe ser superior a 4 para prevenir la diferenciación genética. Si $1<\mathrm{Nm}<0.5$, en ese mismo modelo genético poblacional, la diferenciación genética entre dos poblaciones será leve, pero extremadamente importante en un modelo genético poblacional "stepping-stone". $\mathrm{Si} \mathrm{Nm}<0.5$, las poblaciones consideradas están fuertemente desconectadas para cualquier modelo poblacional. Por lo tanto, los resultados aquí obtenidos muestran claramente que las poblaciones estudiadas están ampliamente desconectadas desde la perspectiva genética.

Relaciones genéticas entre las poblaciones estudiadas: La presencia de 27 especies del 
grupo verrucarum en Colombia y Venezuela, permite sugerir al noroccidente de Suramérica como el lugar de origen del citado grupo. La especiación del grupo parece ser el resultado de diversos eventos biogeográficos y climáticos que han afectado la flora y fauna de la región andina. Una interpretación para tal diversificación, especialmente para la serie townsendi, es el aislamiento de poblaciones ancestrales en refugios de bosque húmedo durante el periodo cuaternario. Cambios climatológicos importantes se dieron durante el Cuaternario (Pleistoceno y Holoceno, 1.6 millones de años hasta el presente). Especialmente importante para la aparición de refugios fueron los ciclos de Milankovitch (cada 19 000-24 000 años, cada 43000 años y cada 90 000-100 000 años durante todo el Cenozoico, incluyendo el Pleistoceno) operando durante el Cuaternario, con fases cálidas y secas alternando con épocas más frías y húmedas (Haffer 1997, Whitmore y Prance 1987).

El origen de la serie townsendi podría estar en un componente de la serie verrucarum ampliamente diseminado, el cual quedó aislado en pequeñas poblaciones en las zonas elevadas de los Andes. Las especies de esta serie en Colombia presentan una distribución con registros puntuales entre las Cordilleras Occidental y Oriental, lo que demuestra una adaptación evolutiva muy específica a las grandes alturas (Bejarano et al. 2003). No es fácil determinar a partir de cuales poblaciones se formaron las del grupo verrucarum. Es posible que ciertas poblaciones hayan experimentado un efecto fundador, con actuación de la deriva genética más intensamente que otras. Los resultados presentes muestran que las especies más relacionadas son $L u$. longiflocosa-Lu. torvida (0.959) у Lu. torvida-Lu. spinicrassa (0.960) mientras que las genéticamente más distantes fueron Lu. torvida-Lu. youngi (0.805) y Lu. quasitownsendi-Lu. youngi (0.796). Los valores de identidad genética obtenidos fueron similares a los encontrados por Kreutzer et al. (1990) $(\mathrm{I}=0.988-0.665)$ para cinco especies de la serie townsendi, presentes en Venezuela y Colombia. Ayala (1975) efectuó estimaciones de la diferenciación genética en los procesos de especiación alopátrica en el grupo de Drosophila willistoni. Las poblaciones locales de una misma especie presentaron un valor promedio de I de $0.970 \pm 0.006$, lo cual es muy similar a las relaciones encontradas entre Lu. longiflocosa-Lu. torvida y Lu. torvidaLu. spinicrassa. Entre subespecies y especies incipientes, los valores fueron muy similares $(0.795 \pm 0.013$ y $0.798 \pm 0.026$, respectivamente) concordando con los valores más elevados encontrados en el presente estudio (Lu. torvida$L u$. youngi y $L u$. quasitownsendi-L. youngi). En ningún caso, las estimas de las identidades genéticas obtenidas fueron tan pequeñas como las encontradas entre especies sinmórficas ( $\mathrm{I}=$ $0.563 \pm 0.023)$ y especies alomórficas $(\mathrm{I}=0.352$ $\pm 0.023)$ en el grupo de $D$. willistoni. No obstante, existen numerosos ejemplos documentados donde la existencia de aislamiento reproductivo ha tenido lugar con mínimos cambios en genes estructurales. Algunos ejemplos son suficientes. D. pseudoobscura del altiplano cundiboyacense colombiano posee un grado de aislamiento reproductivo importante respecto a sus conespecíficos norteamericanos debido tan solo a 4 loci (Prakash 1972). Igualmente, Chrysopa carnea y $C$. downesi (neurópteros) se diferenciaron reproductivamente tan solo por cambios en 3 loci (Tauber y Tauber 1977). Muchas de las más de 500 especies de Drosophila en las islas Hawai, muestran elevadas identidades genéticas entre sí $(\mathrm{I}=0.94-0.99$, Carson et al. 1975, Sene y Carson 1976), lo mismo que formas aisladas reproductivamente de $D$. athabasca $(\mathrm{I}=0.91$, Johnson 1978). Lo mismo habita en otros insectos, como es el caso del lepidóptero Speyeria (5 especies, I = 0.89-0.98, Brittnacher et al. 1978), o, incluso, entre vertebrados, como es el caso de peces del género Cyprinidon (5 especies, I = 0.81-0.97, Turner 1974) o especies de Roedores donde la especiación se da por mecanismos cromosómicos estasipátricos (Spalax ehrenbergi, I $=0.978$, Proechimys guairae, $\mathrm{I}=0.969$, Ayala 1975, Benado et al. 1979). Este podría haber sido el caso entre algunos de los taxones de Lutzomyia estudiados.

El árbol UPGMA con la distancia de Nei mostró que la especie más divergente es 
Lu. youngi, mientras que las relaciones más conspicuas se observan entre Lu. longiflocosaLu. torvida y Lu. torvida-Lu. spinicrassa. La divergencia de $L u$. youngi respecto a las otras especies puede estar dada por un aumento en la deriva genética en el momento de su formación o por la existencia de eventos selectivos sobre uno o varios de los loci polimórficos estudiados que desviaron su perfil genético del resto de las especies analizadas. Sin embargo, la hipótesis de la diferenciación por deriva genética parece más factible ya que $L u$. youngi presentó los niveles promedios de $\mathrm{H}$ más bajos y fue la especie que reveló, en general, menor número de alelos en los loci polimórficos estudiados. La interpretación de este resultado parece favorecer, por lo tanto, la hipótesis de que la aparición del Istmo de panamá pudo haber afectado la distribución de Lu. youngi permitiendo el paso de esta especie entre Sur y Centroamérica (Bejarano et al. 2003).

Autocorrelación Espacial: La mayoría de los alelos utilizados presentaron una estructura espacial muy débil o inexistente, lo que significa que los eventos de especiación entre los taxones estudiados se dieron en forma, más o menos, independiente de las distancias geográficas actuales en donde actualmente se encuentran las cinco especies analizadas. En un contexto de poca autocorrelación espacial, sin embargo, ciertos marcadores mostraron la existencia de autocorrelación espacial significativa. Gpi-B fue el que presentó autocorrelación espacial más conspicua mostrando diferenciación a larga distancia. Es decir, las especies más alejadas (entre 176 y $302 \mathrm{~km}$ ) son también las genéticamente más diferenciadas para este locus. Por lo tanto, este marcador podría ser el que reflejara de una forma más evidente la actuación de la selección natural asociada a diferentes ambientes que, a su vez, se diferenciaron por marcadas distancias geográficas entre ellos. Otros casos de diferenciación genética a larga distancia, pero estrictamente sin alcanzar significación estadística, fueron: 6-pgdh-B, Pgm-B y Est2-C. Estos son, así pues, los marcadores que más han podido verse influidos por procesos de especiación afectados por las distancias geográficas que separaron el origen de esas especies. Es posible que el impacto de la selección natural diversificante haya afectado más a esos marcadores porque estén relacionados con características que dependen fundamentalmente de las diferencias ecológicas en los diversos puntos de muestreo separados geográficamente. Hay otros pocos marcadores que muestran una segunda tendencia espacial diferente: Aat2-C, Idh-A y Est2-E. Los correlogramas de estos marcadores poseen forma de clina circular. Es decir, cuando se formaron las diferentes especies, las diferencias genéticas más grandes se dieron entre los grupos que estaban a una mediana distancia geográfica pero no entre las que presentaron distancias más lejanas desde el punto de vista geográfico.

La existencia de heterogenidad genética significativa (medida por el estadístico $\mathrm{F}_{\mathrm{ST}}$ ), la existencia de una escasa autocorrelación espacial, de un porcentaje significativo de asociación entre las superficies de un cierto grupo de las variables estudiadas, y de la inexistencia de similitud entre los patrones de los correlogramas de los alelos estudiados, nos permiten rechazar ciertos eventos evolutivos como los más probables en la diferenciación genética de las especies estudiadas. La existencia de una moderada estructura espacial, de débil correlación entre las superficies y de débil paralelismo entre los correlogramas permitió proponer al efecto fundador (mecanismo estocástico) como el agente evolutivo más importante en la estructura genética de los humanos de la isla de Bougainville (Sokal y Friedlaender 1982). Los indígenas Yanomami presentaron una fuerte estructura espacial y débil correlación entre las superficies alélicas y débil paralelismo de correlogramas. Deriva genética y fenómenos de fisión-fusión fueron propuestos como los agentes evolutivos básicos que habían operado espacialmente en estos indígenas (Sokal et al. 1986). Para la colonización, por parte de los humanos, de Europa y el Próximo Oriente con el estudio de frecuencias de HLA, se determinó un fuerte porcentaje de casos significativos 
tanto para la estructura espacial, como para la correlación de las superficies y para el paralelismo de los correlogramas. La migración por difusión resultó el evento más probable en la determinación de esas características genéticas (Sokal y Menozzi 1982). Lo mismo ocurrió para una población de caracoles, Helix aspersa (Sokal y Oden 1978a). Drosophila buzzatii, en el este de Australia, mostró una estructura espacial moderada, una débil correlación de superficies y un moderado paralelismo de los correlogramas (con algunas agrupaciones significativas). Selección natural en niveles jerárquicos diferentes fue la explicación invocada para este ejemplo (Sokal et al. 1987). Como en nuestro caso, no se da ninguna de esas combinaciones, todas esas posibles explicaciones evolutivas pueden ser descartadas como los agentes básicos en la diferenciación genética de las especies de Lutzomyia estudiadas aquí. Obviamente, como no se dio homogeneidad estadística para el conjunto de isoenzimas estudiados, explicaciones como idéntica adaptación a los mismos ambientes o poblaciones grandes con elevado flujo génico y panmixia pueden ser inmediatamente descartados. El hecho, que exista una muy limitada cantidad de autocorrelación espacial significativa, muestra que la distancia geográfica entre las especies analizadas no ha jugado un papel demasiado destacado en la diferenciación genética de los loci estudiados. Sin embargo, el hecho que exista heterogeneidad significativa con pérdida de patrones espaciales significativos puede ser explicado, al menos, con dos posibles explicaciones (Sokal y Jacquez 1991). Poblaciones aisladas, sin flujo génico entre ellas, posibilitaría la actuación de la deriva genética en cada localidad, la cual podría ser independiente en magnitud y dirección entre las poblaciones cercanas. Otra posibilidad es la existencia de parches ambientales (con diferente presión de selección natural) dispuestos espacialmente al azar. Sin embargo, podemos distinguir entre esos dos modelos puros examinando la correlación entre las superficies y el paralelismo entre los correlogramas. En el primer modelo, las superficies génicas no deberían estar correlacionadas, ya que la deriva genética habrá actuado de forma diferente en cada locus. En el segundo modelo, se deben encontrar pequeñas y significativas agrupaciones de variables correlacionadas siguiendo una misma presión de selección en los diferentes parches debido a un factor ambiental dado. Este segundo caso, se ajusta perfectamente a los resultados. Como esos factores selectivos solo afectaran de forma idéntica a una pequeña parte de las variables analizadas, la similitud entre los correlogramas será muy baja. Sin embargo, los procesos estocásticos, como la deriva genética, aunque generan diferentes funciones que resultan en patrones independientes de las frecuencias génicas, generan similares correlogramas porque los patrones tienen la misma estructura de varianza-autocovarianza tal como demostraron Sokal y Wartenberg (1983). Es decir, si los fenómenos de aislamiento por distancia y deriva genética fueran fuertes, esperaríamos un porcentaje elevado de similares formas en los correlogramas, fenómeno no observado en los presentes datos. Por lo tanto, podemos concluir que, en conjunto, para las variables analizadas, los fenómenos de diferenciación genética (y, quizás, de especiación) entre las especies consideradas han sido más afectados por la selección natural dependiente de parches ambientales dispuestos al azar con actuación en ciertos alelos que por la acción de los procesos estocásticos, como la deriva genética, que permiten la diferenciación por distancia.

Futuros estudios moleculares deberán revelar una relación más precisa entre los cambios climáticos y geológicos del Cuaternario y la filogeografía y fuerte especiación de Lutzomyia en los Andes sudamericanos.

\section{AGRADECIMIENTOS}

Al Instituto Nacional de Salud y a Colciencias por la ayuda financiera que permitió la realización de este estudio. A Olga Lucía Cabrera (Laboratorio de Entomología, Instituto Nacional de Salud) por su colaboración en la colonización de las especies de Lutzomyia en el laboratorio. A la Pontificia Universidad 
Javeriana por brindar la capacidad computacional para llevar a cabo los análisis genético poblacionales. A Diana Alvarez por su ayuda en la realización de las figuras y tablas.

\section{RESUMEN}

Se analizaron 16 sistemas isoenzimáticos para cinco especies colombianas del género Lutzomyia. Los niveles de heterocigosis media esperada insesgada oscilaron entre 0.098 (Lu. youngi) y 0.215 (Lu. torvida). Las cinco muestras estudiadas de forma global, para todos los marcadores analizados, presentaron desviación respecto al equilibrio Hardy-Weinberg por un exceso de homocigotos, tanto al utilizar algunas pruebas clásicas como tests exactos con cadenas de Markov. Este hecho puede estar favorecido por diversas causas: (1) la más probable es la existencia de efecto Wahlund en el seno de cada población debido a subdivisión y/o a la técnica de muestreo empleada. La endogamia puede descartarse ya que no todos los loci están afectados por el mismo tipo de exceso de homocigotos. (2) Sin embargo, no se puede descartar la existencia de alelos nulos, al menos, para algunos de los marcadores isoenzimáticos utilizados. El análisis jerarquizado con las F de Wright mostró valores elevados y significativos para cada uno de los estadísticos. El estadístico promedio $\mathrm{F}_{\text {IT }}$ mostró un valor de 0.655 existiendo un conspicuo exceso de homocigotos a nivel total de todas las especies, el estadístico promedio $\mathrm{F}_{\mathrm{IS}}$ fue altamente positivo (0.515) mostrando exceso de homocigotos a nivel individual en cada una de las especies estudiadas. La heterogeneidad genética entre las cinco especies fue notable $\left(\mathrm{F}_{\mathrm{ST}}=0.288\right)$. Esto muestra que esas especies están bien diferenciadas a nivel isoenzimático y que en el interior de cada especie también hay una subdivisión genética. La matriz de identidades genéticas de Nei muestra que las especies más relacionadas fueron $\mathrm{Lu}$. longiflocosa-Lu. torvida (0.959) y $L u$ torvida-Lu. spinicrassa (0.960) mientras que las genéticamente más distantes fueron $L u$. torvida-Lu. youngi (0.805) y Lu. quasitownsendi-Lu. youngi (0.796). Con los algoritmos UPGMA y Wagner, se observó que la especie más divergente fue $L u$. youngi, mientras que las relaciones más conspicuas se observaron entre $L u$. longiflocosa- $L u$. torvida y Lu torvida-Lu. spinicrassa. Adicionalmente, con un análisis de autocorrelación espacial (índice de Moran) la mayoría de los alelos utilizados presentaron una estructura espacial muy débil o inexistente, lo que significa que los eventos de especiación entre las especies estudiadas se dieron en forma independiente de las distancias geográficas existentes actualmente entre ellas.

Palabras Clave: Isoenzimas, genética de poblaciones, Lutzomyia, grupo verrucarum, Colombia.

\section{REFERENCIAS}

Adamson, R.E., M. L. Chance, R. D. Ward, D. Feliciangeli, \& R. Maigon. 1991. Molecular approaches applied to the analysis of sympatric sand fly populations in endemic areas of Western Venezuela. Parassitologia 33 (Supp. 1): 45-53.

Adamson, R.E., R. D. Ward, D. Feliciangeli, \& R. Maigon. 1993. The aplication of random amplified polimorphic DNA for sandfly species identification. Med. Vet. Entomol. 7: 203-207.

Añez, N., D. T. Valenta, D. Cazorla, D. J. Quicke, \& M.D. Felinciangeli. 1997. Multivariate analysis to discriminate species of phlebotomine sand flies (Diptera: Psychodidae): Lutzomyia townsewndi, L. spinicrassa and L. youngi. J. Med. Entomol. 34: 312-316.

Ayala, F.J. 1975. Genetic differentiation during the speciation process. Evol. Biol. 8: 1-78.

Bejarano, E., W. Rojas, S. Uribe, \& I. D. Vélez. 2003. Sistemática de especies del grupo verrucarum Theodor, 1965 (Diptera: Psychodidae). Biomédica 23: $87-102$.

Benado, M., M. Aguilera, O. A. Reig, \& F. J. Ayala. 1979. Biochemical genetics of chromosome forms of Venezuelan spiny rats of the Proechimys guairae and Proechimys trinitatis superspecies. Genetica 50: 89-97.

Brittnacher, J.C., S. R. Sims, \& F. J. Ayala. 1978. Genetic differentiation between species of the genus Speyeria (Lepidoptera: Nymphalidae). Evolution 32: 199-210.

Carson, H.L., W. E. Jonson, P. S. Nair, \& F. M. Sene. 1975. Allozymic and chromosomal similarity in two Drosophila species. Proc. Nat. Acad. Sci. U.S.A. 72: 4521-4525.

Cavalli-Sforza, L.L., \&A.W.F. Edwards. 1967. Phylogenetic analysis: models and estimation procedures. Evolution 21: $550-570$.

Dobzhansky, Th. 1971. Evolutionary oscillations in Drosophila pseudoobscura. In: Ecological genetics and Evolution. Pp: 109-133. Blakwell Scientific, Oxford, Reino Unido.

Endris, R.G., P. V. Perkins, D. G. Young, \& R. N. Johnson. 1982. Techniques for laboratory rearing of sand flies (Diptera: Psychodidae). Mosquito News 42: 400-407. 
Escovar, J. 2000. Comparación cariotípica de cinco especies de Lutzomyia correspondientes al grupo verrucarum y a la serie townsendi. Tesis de Licenciatura en Química y Biología. Universidad de La Salle, Facultad de Ciencias de La Educación. 101 Pp. Bogotá, Colombia.

Feliciangeli, M.D, \& J. Murillo. 1987. Lutzomyia youngi (Diptera: Psychodidae), a new phlebotomine sand fly previously misidentified as $L$. townsendi in endemic foci of cutaneous leishmaniasis in Venezuela and Costa Rica. J. Med. Entomol. 24: 141-146.

Feliciangeli, M.D., O. C. Castejon, \& J. Limongi. 1993. Egg surface ultraestructure of eight New World phlebotomine sandfly species (Diptera: Psychodidae). J. Med. Entomol. 30: 651-656

Gabriel, K.R., \& R.R. Sokal. 1969. A new statistical approach to geographic variation analysis. Systematic Zoology 18: 259-278.

Gower, J.C., \& G. J. S. Ross. 1969. Minimum spanning trees and single cluster analysis. Appl. Stat. 18: 54-64.

Haffer, J. 1997. Alternative models of vertebrate speciation in Amazonia: an overview. Biodiversity Conservation 6: $451-476$

Johnson, D.E.L. 1978. Genetic differentiation in two members of the Drosophila athabasca complex. Evolution 32: 798-811.

Killick-Kendrick, R. 1990. Phlebotomine vectors of the leishmaniases: a review. Med. Vet. Entomol. 4: 1-24.

Kreutzer, R.D., M. T. Palau, A. Morales, C. Ferro, D. Feliciangeli, \& D. G. Young. 1990. Genetic relationships among phlebotomine sand flies (Diptera: Psychodidae). J. Med. Entomol 27: 1-8.

Kruskal, J.B. 1964a. Multidimensional scaling by optimizing goodness of fit to a nonmetric hypthesis. Psychometrika 29: 1-27.

Kruskal, J.B. 1964b. Nonmetric multidimensional scaling: a numerical method. Psychometrika 29: 28-42.

Lainson, R. 1983. The American leishmaniasis: some observations on their ecology and epidemiology. Trans Royal. Soc. Trop. Med. Hyg. 77: 569-596.

Maigon, R., D. Feliciangeli, R. Ward, M. Chance, R. Adamson, N. Rodriguez, J. Convit, J. Petralanda, A. Hernández, \& M. Segovia. 1993. Molecular approaches applied to the epidemiology of Leishmania in Venezuela. Arch. Inst. Pasteur. Tunis 70: 309-324.
Matula, D.W., \& R. R. Sokal. 1980. Properties of Gabriel graphs relevant to geographic variation research and the clustering of points in the plane. Geogr. Anal. 12: 205-222.

Nei, M. 1972. Genetic distance between populations. Amer. Nat. 106: 283-292.

Nei, M. 1978. Estimation of average heterozygosity and genetic distance from a small number of individuals. Genetics 89: 583-590.

Oden, N.L. 1984. Assessing the significance of a spatial correlogram. Geogr.. Anal. 16: 1-16.

Pérez, S.P. 1996. Diferenciación morfométrica de las hembras de la serie townsendi del grupo verrucarum, género Lutzomyia presentes en Colombia en los focos endémicos de leishmaniasis cutánea. Tesis de Licenciatura en Química y Biología. Universidad de La Salle, Facultad de Ciencias de La Educación. 108 Pp. Bogotá, Colombia.

Prakash S. 1972. Origin of reproductive isolation in the absence of apparent genic differentiation in a geographic isolate of Drosophila pseudoobscura. Genetics 72: 143-155.

Robertson, A., \& W. G. Hill. 1984. Deviations from HardyWeinberg proportions, sampling variances and use in estimation of inbreeding coefficients. Genetics 107: 703-718.

Rojas, W., S. Uribe, E. Bejarano, C. Porter, \& I. D. Vélez. 2000. Molecular search of markers for the diagnosis of species of Lutzomyia spp. of the verrucarum group (Theodor, 1965). Abstracts, Vol. 2. XV International Congress for Tropical Medicine and Malaria. Pp. 131. Cartagena de Indias, Colombia.

Sene, F.M., \& H. R. Carson. 1976. Close allozymic similarity within and between two sympatric species of Hawaiian Drosophila. Genetics 83: 269-270.

Sierra, D., I. D. Vélez, \& S. Uribe. 2000. Identificación de Lutzomyia spp. (Diptera: Psychodidae) grupo verrucarum por medio de microscopia electrónica de sus huevos. Rev. Biol. Trop 48: 615-622.

Slatkin, M. 1985. Rare alleles as indicators of gene flow. Evolution 39: 53-65.

Sokal, R.R. 1986. The spatial analysis of human population structure. Symposia Thrakika 1984: 1-15.

Sokal, R.R., \& J. Friedlaender. 1982. Spatial autocorrelation analysis of biological variation on Bougainville Island. Pages 205-277. In M. H. Crawford and J. H. Mielke (Eds). Current developments in anthropological genetics. Vol. 2. Plenum, Nueva York, EEUU. 
Sokal, R. R., \& G. M. Jacquez. 1991. Testing inferences about microevolutionary proceses by means of spatial autocorrelation analysis. Evolution 45: 152-168.

Sokal, R.R., \& P. Menozzi. 1982. Spatial autocorrelation of HLA frequencies in Europe support demic difusión of early farmers. The American Naturalist 119: 1-17.

Sokal, R.R., \& N. L. Oden. 1978a. Spatial autocorrelation in biology. 1. Methodology. Biol.. J. Linn. Soc. 10: 199-228.

Sokal, R.R., \& N. L. Oden. 1978b. Spatial autocorrelation in biology. 2. Some biological implications and four applications of evolutionary and ecological interest. Biol. J. Linn. Soc. 10: 229-249.

Sokal, R.R., \& D.E. Wartenberg. 1983. A test of spatial autocorrelation using an isolation-by-distance model. Genetics 105: 219-237.

Sokal, R.R., R. M. Harding, \& N. L. Oden. 1989. Spatial patterns of human gene frequencies in Europe. Amer. J. Phys. Anthropol. 80: 267-294.

Sokal, R.R, N. L. Oden, \& J. S. F. Barker. 1987. Spatial structure in Drosophila buzzattii populations: simple and directional spatial autocorrelation. The American Naturalist 129: 122-142.

Sokal, R.R., P. E. Smouse, \& J. V. Neel. 1986. The genetic structure of a tribal popualtion, the Yanomama Indians genetics. XV. Patterns inferred by autocorrelation analysis. Genetics 114: 259-287.

Spath, H. 1983. Cluster-Formation und Analyse. R. Oldenboug, Munich, Alemania.
Tauber, C., \& M. Tauber. 1977. Sympatric speciation based on allelic changes at three loci. Evidence from natural populations in two habitats. Science 197: 1298-1299.

Turner, B.J. 1974. Genetic divergence of Death Valley pup fish species: Biochemical versus morphological evidence. Evolution 28: 281-294.

Valenta, D.T., N. Añez, Y. Tang, R. Killick-Kendrick. 1999. The genital atrium as a good taxonomic character to distinguish between species of phlebotomine sandflies (Diptera: Psychodidae) from Venezuela. Ann. Trop. Med. Parasitol 93: 389-399.

Whitmore, T. C., \& G. T. Prance. 1987. Biogeography and Quaternary History in Tropical America. In: Oxford Monographs in Biogeography, vol. 3. Oxford University, Oxford, Reino Unido.

Wright, S. 1931. Evolution in Mendelian populations. Genetics 16: 97-159.

Wright, S. 1943. Isolation by distance. Genetics 28: 114138.

Young, D.G. 1979. A review of the blood sucking Psychodidae flies of Colombia (Diptera: Phlebotominae and Sycoracinae). Tech. Bull 806, Institute of Food and Agricultural Sciences. Pp 1-265. Gainsville, Florida, EEUU.

Young, D.G., \& M. A. Duncan. 1994. Guide to the identification and geographic distribution of Lutzomyia sand flies in Mexico, the West Indies, Central and South America (Diptera: Psychodidae). Associated Publishers American Entomological Institute, Florida, EEUU. 
